\title{
Atuação do Cirurgião-Dentista na oferta de cuidados bucais em pacientes da terceira idade: uma revisão de literatura
}

\author{
Performance of the dentist in the provision of oral care to elderly patients: a literature review \\ El papel de los dentistas em el cuidado bucal de los pacientes ancianos: revisión de la literatura
}

Recebido: 24/09/2021 | Revisado: 30/09/2021 | Aceito: 04/10/2021 | Publicado: 05/10/2021

\author{
Mikelle Araújo Gomes \\ ORCID: https://orcid.org/0000-0001-5531-6692 \\ Universidade Estadual da Paraíba, Brasil \\ E-mail: mikelle93@hotmail.com \\ Natan da Silva Oliveira \\ ORCID: https://orcid.org/0000-0002-9207-2428 \\ Universidade Estadual da Paraíba, Brasil \\ E-mail: natan150399@gmail.com \\ Natália Medeiros Andrade \\ ORCID: https://orcid.org/0000-0003-1059-8205 \\ Universidade Estadual da Paraíba, Brasil \\ E-mail: nataliama16@hotmail.com \\ Mateus Ferreira de Farias \\ ORCID: https://orcid.org/0000-0001-8184-7307 \\ Universidade Estadual da Paraíba, Brasil \\ E-mail: mateusuepb@outlook.com \\ Laís Gonzaga de Farias \\ ORCID: https://orcid.org/0000-0001-9362-7774 \\ Universidade Estadual da Paraíba, Brasil \\ E-mail: lais.gonzagaa@gmail.com \\ Maria Helena Chaves de Vasconcelos Catão \\ ORCID: https://orcid.org/0000-0001-7681-3225 \\ Universidade Estadual da Paraíba, Brasil \\ E-mail:mhcvcatao@gmail.com
}

\begin{abstract}
Resumo
Os problemas bucais possuem efeito negativo na saúde geral e na qualidade de vida dos idosos, dessa forma, é imprescindível que os sistemas de saúde bucal garantam a saúde oral de todas as pessoas da terceira idade. O presente artigo tem como objetivo relatar a atuação do Cirurgião-Dentista na oferta de cuidados bucais em pacientes da terceira idade. Tratou-se de uma revisão integrativa de literatura por meio da busca bibliográfica nas bases de dados BVS e PubMed. Foram incluídos artigos nos últimos 10 anos (2011 a 2021) e que possuíam o texto completo e, excluídos, os que não abordavam a temática principal da presente pesquisa e os quais não foi possível o acesso ao texto completo. Inicialmente, 120 artigos foram identificados na BVS e PubMed por meio da busca por descritores. Na base de dados PubMed, após a aplicação dos filtros, foram encontrados 09 resultados. Destes, 06 artigos foram selecionados e 01 excluído por não ser possível obter acesso ao texto completo. Já na base de dados BVS, após a aplicação dos filtros, foram encontrados 03 resultados. Destes, após a leitura do título e resumo, 02 artigos foram selecionados, e os 02 foram excluídos por não obter acesso ao texto completo. Dessa forma, totalizaram-se 05 publicações disponíveis, as quais foram incluídas nesse estudo. Conclui-se que a atuação do cirurgião-dentista se faz extremamente importante nos cuidados bucais de pacientes da terceira idade.
\end{abstract}

Palavras-chave: Odontologia geriátrica; Serviços de saúde bucal; Saúde bucal; Idoso; Odontólogos.

\begin{abstract}
Oral problems have a negative effect on the general health and quality of life of the elderly, thus, it is essential that oral health systems ensure the oral health of all elderly people. This article aims to report the role of dentists in providing oral care to elderly patients. This was an integrative literature review through a bibliographic search in the BVS and PubMed databases. Articles in the last 10 years (2011 to 2021) that had the full text were included, and those that did not address the main theme of this research and those that did not have access to the full text were excluded. Initially, 120 articles were identified in the VHL and PubMed through the search for descriptors. In the PubMed database, after applying the filters, 09 results were found. Of these, 06 articles were selected and 01 excluded because it was not possible to obtain access to the full text. In the VHL database, after applying the filters, 03 results were found. Of these, after reading the title and abstract, 02 articles were selected, and the 02 were excluded for not having
\end{abstract}


access to the full text. Thus, a total of 05 publications were available, which were included in this study. It is concluded that the role of the dentist is extremely important in the oral care of elderly patients.

Keywords: Geriatric dentistry; Dental health services; Oral health; Aged; Dentists.

\section{Resumen}

Los problemas bucales tienen un efecto negativo sobre la salud general y la calidad de vida de las personas mayores, por lo que es fundamental que los sistemas de salud bucal garanticen la salud bucal de todas las personas mayores. Este artículo tiene como objetivo informar sobre el papel de los dentistas en la prestación de cuidados bucales a los pacientes de edad avanzada. Se trató de una revisión integradora de la literatura mediante una búsqueda bibliográfica en las bases de datos BVS y PubMed. Se incluyeron artículos de los últimos 10 años (2011 a 2021) que tenían el texto completo, y se excluyeron aquellos que no abordaban el tema principal de esta investigación y aquellos que no tenían acceso al texto completo. Inicialmente, se identificaron 120 artículos en la BVS y PubMed mediante la búsqueda de descriptores. En la base de datos de PubMed, luego de aplicar los filtros, se encontraron 09 resultados. De estos, se seleccionaron 06 artículos y se excluyeron 01 porque no fue posible obtener acceso al texto completo. En la base de datos de BVS, luego de aplicar los filtros, se encontraron 03 resultados. De estos, luego de leer el título y el resumen, se seleccionaron 02 artículos, y se excluyeron los 02 por no tener acceso al texto completo. Así, se dispuso de un total de 05 publicaciones, que fueron incluidas en este estudio. Se concluye que el papel del odontólogo es de suma importancia en el cuidado bucal de los pacientes ancianos.

Palabras clave: Odontología geriátrica; Servicios de salud bucal; Salud bucal; Anciano; Dentistas.

\section{Introdução}

O desenvolvimento socioeconômico na maioria das regiões foi acompanhado por grandes reduções na fertilidade atrelado a grandes aumentos na expectativa de vida nos últimos 50 anos. Assim, como resultado, obteve-se rápidas mudanças na demografia das populações no mundo todo. De modo que, a proporção de idosos na população em geral aumentou consideravelmente dentro de um curto período de tempo (World Health Organization., 2015). A proporção da população com 60 anos ou mais quase dobrará em 2050 no mundo, já nas Américas, a expectativa de vida aumentou 21,6 anos nas últimas seis décadas (World Health Organization., 2017).

O envelhecimento populacional é um fenômeno já evidente no Brasil e nas próximas décadas tende a ficar ainda mais marcante, sendo caracterizado pelo aumento da participação percentual dos idosos na população e consequente diminuição dos demais grupos etários (Instituto Brasileiro De Geografia E Estatística [IBGE], 2015). De acordo com A Projeção da População por Sexo e Idade, realizada pelo IBGE (2013), foi observado uma tendência de aumento da proporção de idosos na população. De maneira que em 2030, esta proporção seria de 18,6\%, e, em 2060, de 33,7\%, ou seja, a cada três pessoas na população uma terá ao menos 60 anos de idade. A transição demográfica afetará quase todos os aspectos da sociedade, de maneira que crie novos desafios para os sistemas de saúde e assistência social (World Health Organization, 2017).

No Estatuto do Idoso é assegurado às pessoas de 60 anos ou mais a efetivação do direito à liberdade, à dignidade e ao respeito, saúde, educação, cultura, esporte e lazer, profissionalização e trabalho, previdência social, de forma geral, à vida (Brasil, 2013).

Com o aumento da idade, o risco de doenças e deficiências consequentemente aumentam. Enfrentar uma população de idosos tão numerosa, com problemas de saúde que afetam sua qualidade de vida, não só a sua saúde física ou psicológica tornou-se um grande problema de saúde pública. Outrossim, existe uma relação complexa e mútua entre saúde geral e saúde oral. De modo que doenças sistêmicas podem aumentar o risco de doenças bucais e vice-versa. Sendo assim, a saúde oral faz parte da saúde geral e contribui para o bem-estar físico, psicológico e social de uma pesso. (Ástvaldsdóttir et al., 2018; Glick et al., 2016; Lyu \& Wolinsky, 2017).

A doença oral em idosos não só causa dor, diminuição da capacidade mastigatória e alterações na fala e aparência física, como também afeta a saúde geral e aumenta o risco de doenças neurológicas, endócrinas, cardiovasculares e respiratórias. Problemas de saúde bucal estão associados a mudanças na seleção de alimentos, diminuição da qualidade 
nutricional. Tendo assim, efeito negativo na saúde geral e na qualidade de vida, e podendo limitar as interações sociais (Castrejón-pérez et al., 2017; Donnelly et al., 2016; Masood et al., 2017).

A Odontologia geriátrica abrange todos os aspectos da saúde bucal e cuidados bucais de pessoas idosas (Glick et al., 2016). Com isso, os sistemas de saúde bucal devem e podem ser transformados para garantir a saúde oral de todos os idosos. Haja vista que esse foco voltado para atenção à saúde oral dos idosos beneficia não apenas os idosos hoje, mas também os idosos no futuro (Raphael, 2017). Ademais, é necessário contribuir com políticas de saúde que tenham abordagem transformadora na maneira como os serviços de saúde são estruturados, visando garantir cuidados integrados de alta qualidade, acessíveis e com foco nas necessidades e direitos das pessoas idosas. De modo que possibilite antever os cuidados necessários para um envelhecimento saudável(P. Alves et al., 2017; World Health Organization., 2017).

Nesse sentido, o objetivo desta pesquisa é relatar, através da literatura, a atuação do Cirurgião-Dentista na oferta de cuidados bucais em pacientes da terceira idade.

\section{Metodologia}

Trata-se de uma revisão integrativa de literatura, que permitiu a reflexão e compreensão com base em evidências científicas sobre o estudo em questão, com temática voltada a oferta de cuidados bucais em pacientes da terceira idade (Hopia, Latvala \& Liimatainer, 2016).

A pesquisa foi realizada por meio da busca bibliográfica nas bases de dados BVS e PubMed. As publicações indexadas nas bases de dados foram identificadas por meio dos seguintes descritores: "odontologia geriátrica", "serviços de saúde bucal", "saúde bucal”, "idoso" e "odontólogos", e dos respectivos termos em inglês: "geriatric dentistry", "dental health services", "oral health", "aged" e "dentists". Todos os descritores utilizados estão presentes no "Descritores em Ciências da Saúde" (DeCS/MeSH).

Foram incluídos nesse estudo artigos científicos originais presentes nas plataformas de buscas científicas PubMed e BVS publicados nos últimos 10 anos (2011 a 2021) e que possuíam o texto completo. Foram excluídos estudos que não abordavam a temática principal da presente pesquisa e que não foi possível obter acesso ao texto completo.

Os dados foram organizados a partir da utilização do Microsoft Word for Windows e expressos através de fluxograma, quadro de amostragem e um quadro sinóptico contemplando: título do estudo, nome dos autores, ano de publicação, idioma, país de origem, objetivos, método empregado, os principais resultados e conclusões.

\section{Resultados}

A Figura 1 e o Quadro 1, apresentam o processo de seleção dos trabalhos incluídos no estudo. Inicialmente, 120 artigos foram identificados na BVS e PubMed por meio da busca por descritores. 
Figura 1 - Fluxograma de amostragem da revisão de literatura.
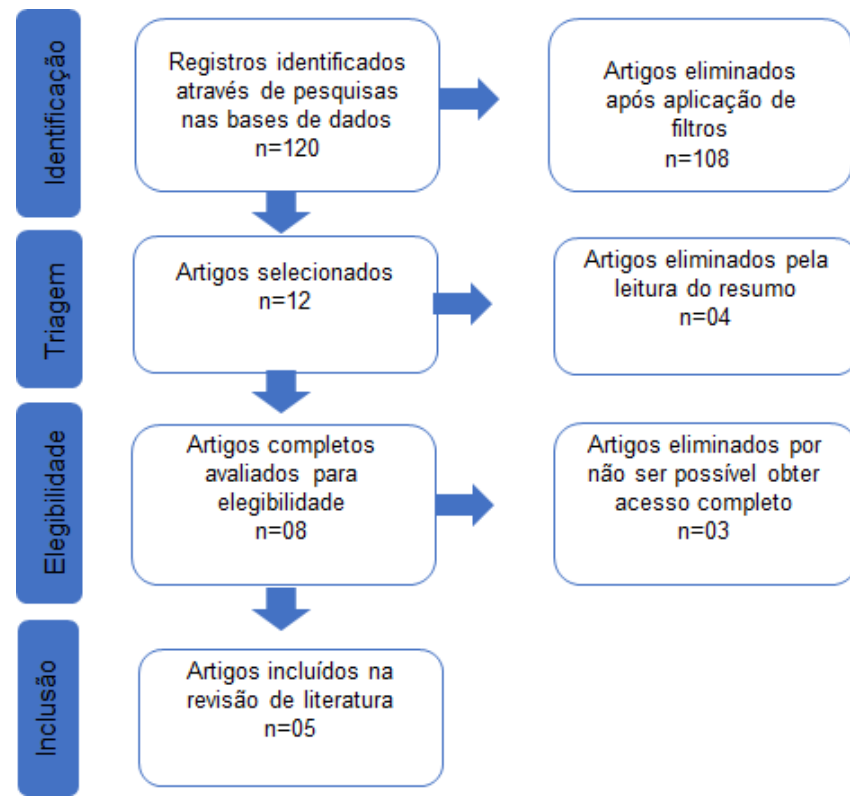

Artigos eliminados por não ser possivel obter acesso completo $n=03$

Fonte: Pesquisa direta (2021).

Quadro 1 - Quadro de amostragem da revisão de literatura.

\begin{tabular}{|c|c|c|c|c|c|c|}
\hline $\begin{array}{c}\text { Base de } \\
\text { dados }\end{array}$ & Descritores & Quantidade & Filtros & Resultado & Exclusões & Resultados \\
\hline BVS & $\begin{array}{c}\text { “odontologia geriátrica", } \\
\text { "serviços de saúde bucal", } \\
\text { "saúde bucal", "idoso" e } \\
\text { "odontólogos" }\end{array}$ & 08 & $\begin{array}{l}\text { "Texto completo } \\
\text { disponível" e } \\
\text { "Publicação nos } \\
\text { últimos } 10 \text { anos" }\end{array}$ & 03 & 03 & $\mathbf{0}$ \\
\hline PubMed & $\begin{array}{l}\text { "geriatric dentristy", "dental } \\
\text { health services", "oral } \\
\text { health", "aged" e "dentists" }\end{array}$ & 112 & $\begin{array}{l}\text { "Texto completo } \\
\text { disponível" e } \\
\text { "Publicação nos } \\
\text { últimos } 10 \text { anos" }\end{array}$ & 09 & 04 & 05 \\
\hline Total & - & 120 & - & 12 & 07 & 05 \\
\hline
\end{tabular}

Fonte: Pesquisa direta (2021).

Na base de dados PubMed, foram encontrados inicialmente 112 resultados para os descritores utilizados. Após a aplicação do filtro de "texto completo disponível”, foram encontrados 12 resultados, na associação com o filtro de publicação nos "últimos 10 anos" foram encontrados 09 resultados e com a leitura do título e resumo, 06 artigos foram selecionados, destes, 01 foi excluído por não ser possível obter acesso ao texto completo.

Já na base de dados BVS, foram encontrados inicialmente 08 resultados para os descritores utilizados. Após a aplicação do filtro de "texto completo disponível”, encontramos 03 resultados. Associado com o filtro de publicação "nos últimos 10 anos", permaneceram os 03 resultados, na leitura do título e resumo, 02 artigos foram selecionados, destes, 02 foram excluídos por não ser possível obter acesso ao texto completo. Dessa forma, totalizaram-se 05 publicações disponíveis, as quais foram incluídas nesse estudo. 


\section{Análise Descritiva dos Estudos}

Com o propósito de sistematizar os dados destes artigos, foi utilizado um instrumento de coleta do Quadro 2, denominado quadro sinóptico, contemplando: título do estudo, nome dos autores, ano de publicação, idioma, país de origem, objetivos, método empregado e os principais resultados e conclusões, para assim, discuti-las de forma detalhada.

Quadro 2 - Análise descritiva dos artigos eleitos.

\begin{tabular}{|c|c|c|c|c|}
\hline $\begin{array}{c}\text { Título/Autores/Ano } \\
\text { Publicação }\end{array}$ & $\begin{array}{c}\text { Idioma } \\
\text { publicado } \\
\text { /País }\end{array}$ & Objetivo & Método & $\begin{array}{c}\text { Principais resultados/ } \\
\text { Conclusões }\end{array}$ \\
\hline $\begin{array}{l}\text { Oral Health-Related } \\
\text { Quality of Life in } \\
\text { institutionalized elderly in } \\
\text { Barcelona (Spain), } \\
\text { Cornejo et al.,2013 }\end{array}$ & $\begin{array}{l}\text { Inglês/ } \\
\text { Espanha }\end{array}$ & $\begin{array}{l}\text { Avaliar o estado de saúde } \\
\text { bucal e os fatores } \\
\text { associados à qualidade de } \\
\text { vida relacionada à saúde } \\
\text { bucal usando GOHAI e } \\
\text { vários fatores que afetam } \\
\text { pacientes idosos } \\
\text { institucionalizadas em } \\
\text { Barcelona em 2009. }\end{array}$ & $\begin{array}{l}\text { Delineamento: estudo } \\
\text { transversal. } \\
\text { Foram realizados em } 194 \\
\text { idosos de } 65 \text { anos ou mais } \\
\text { exames bucais que } \\
\text { forneceram informações } \\
\text { sobre a saúde bucal clínica e } \\
\text { entrevistas para obter dados } \\
\text { sobre questões } \\
\text { sociodemográficas, } \\
\text { autorrelato de saúde bucal e } \\
\text { utilização de serviços } \\
\text { odontológicos. }\end{array}$ & $\begin{array}{l}\text { Observou-se que } 94 \text { mulheres } \\
(68,1 \%) \text { e } 36 \text { homens }(64,3 \%) \\
\text { apresentaram baixo (OHRQoL). } \\
\text { O índice CPOD médio foi de } \\
22,8 \text {, com média de } 10,2 \text { dentes } \\
\text { restantes. De acordo com o } \\
\text { Índice Periodontal Comunitário, } \\
\text { apenas 1,9\% eram saudáveis, } \\
33,8 \% \text { apresentavam } \\
\text { edentulismo, } 54,2 \% \\
\text { necessitavam de próteses } \\
\text { dentárias superiores. Apenas } \\
7,2 \% \text { haviam visitado o dentista } \\
\text { no último ano. A população do } \\
\text { estudo apresenta saúde bucal } \\
\text { objetiva ruim. }\end{array}$ \\
\hline $\begin{array}{l}\text { Oral health status and } \\
\text { need for oral care of care- } \\
\text { dependent indwelling } \\
\text { elderly: from admission } \\
\text { to death, Hoeksema et al., } \\
2017\end{array}$ & $\begin{array}{l}\text { Inglês/ } \\
\text { Holanda }\end{array}$ & $\begin{array}{l}\text { Avaliar a saúde bucal e o } \\
\text { estado bucal de pacientes } \\
\text { idosos recém-admitidos } \\
\text { em uma casa de repouso } \\
\text { desde a admissão até o } \\
\text { óbito. }\end{array}$ & $\begin{array}{l}\text { Delineamento: estudo } \\
\text { longitudinal. } \\
\text { Foi avaliada, a saúde bucal, } \\
\text { estado bucal, necessidade de } \\
\text { atendimento odontológico, } \\
\text { cooperação com tratamento } \\
\text { odontológico e atendimento } \\
\text { odontológico de } 725 \text { idosos } \\
\text { (79 pacientes com demência, } \\
246 \text { pacientes somáticos) } \\
\text { internados em uma casa de } \\
\text { repouso entre janeiro de } \\
2009 \text { e dezembro de } 2013 \text {. }\end{array}$ & $\begin{array}{l}\text { Os pacientes com demência eram } \\
\text { significativamente mais velhos } \\
\text { do que os somáticos. Os } \\
\text { pacientes edêntulos eram } \\
\text { significativamente mais velhos } \\
\text { do que os pacientes com dentes } \\
\text { remanescentes, } 30 \% \text { dos } \\
\text { pacientes internados morreram } \\
\text { dentro de } 12 \text { meses após a } \\
\text { admissão. Uma pequena minoria } \\
\text { (20\%) dos pacientes possuía } \\
\text { dentes próprios. Nesse grupo, } \\
\text { higiene bucal precária (72\%), } \\
\text { cárie (70\%) e dentes quebrados } \\
\text { (62\%) foram frequentemente } \\
\text { observados. racientes } \\
\text { desdentados rosam } \\
\text { significativamente } \\
\text { cooperativos com o tratamento } \\
\text { do que pacientes com dentes } \\
\text { remanescentes. O menor tempo } \\
\text { de atendimento odontológico } \\
\text { profissional foi dado a pacientes } \\
\text { desdentados. }\end{array}$ \\
\hline
\end{tabular}




\begin{tabular}{|c|c|c|c|c|}
\hline $\begin{array}{l}\text { "You Get Beautiful Teeth } \\
\text { Down There": } \\
\text { Racial/Ethnic Minority } \\
\text { Older Adults' } \\
\text { Perspectives on Care at } \\
\text { Dental School Clinics, } \\
\text { Northridge et al., } 2017\end{array}$ & $\begin{array}{l}\text { Inglês/Estados } \\
\text { Unidos }\end{array}$ & $\begin{array}{l}\text { Examinar as experiências } \\
\text { de idosos afro- } \\
\text { americanos, porto- } \\
\text { riquenhos e dominicanos } \\
\text { que frequentam centros } \\
\text { para idosos em } \\
\text { Manhattan, na cidade de } \\
\text { Nova York, em relação ao } \\
\text { atendimento recebido em } \\
\text { clínicas de escolas de } \\
\text { odontologia. }\end{array}$ & $\begin{array}{l}\text { Delineamento: estudo } \\
\text { qualitativo. Foram realizadas } \\
\text { de } 2013 \text { a 2015, } 24 \text { sessões } \\
\text { de grupo, com uma amostra } \\
\text { de } 194 \text { homens e mulheres } \\
\text { de minorias raciais/ étnicas } \\
\text { com } 50 \text { anos ou mais que } \\
\text { viviam na parte alta de } \\
\text { Manhattan, em que foram } \\
\text { gravadas em áudio digital e } \\
\text { transcritas para análise. }\end{array}$ & $\begin{array}{l}\text { A maioria dos participantes } \\
\text { relatou experiências positivas } \\
\text { com clínicas de escolas de } \\
\text { odontologia. Apesar de algumas } \\
\text { opiniões negativas, elas não } \\
\text { foram expressas universalmente } \\
\text { pelos participantes. } \\
\text { Este estudo adiciona contexto } \\
\text { aos esforços das escolas de } \\
\text { odontologia para enfrentar os } \\
\text { desafios de cuidar da cada vez } \\
\text { mais diversificada população } \\
\text { idosa. Para eliminar as } \\
\text { desigualdades raciais, étnicas e } \\
\text { socioeconômicas relatadas no } \\
\text { tratamento em escolas de } \\
\text { odontologia. }\end{array}$ \\
\hline $\begin{array}{l}\text { Oral health-related } \\
\text { quality of life among } \\
\text { elderly patients visiting } \\
\text { special clinics in public } \\
\text { hospitals in Delhi, India: } \\
\text { A cross-sectional study, } \\
\text { Chahar; Mohanty; } \\
\text { Aswini, } 2019\end{array}$ & Inglês/Índia & $\begin{array}{c}\text { Avaliar a qualidade de } \\
\text { vida relacionada à saúde } \\
\text { bucal (OHRQoL) usando } \\
\text { GOHAI e vários fatores } \\
\text { que afetam pacientes } \\
\text { idosos que visitam } \\
\text { clínicas geriátricas } \\
\text { especiais aos domingos } \\
\text { em hospitais públicos de } \\
\text { Delhi. }\end{array}$ & $\begin{array}{l}\text { Delineamento: estudo } \\
\text { transversal. Foram coletados } \\
\text { por conveniência uma } \\
\text { amostra de } 145 \text { pacientes } \\
\text { idosos. O (OHRQoL) foi } \\
\text { medido usando o } \\
\text { instrumento pré-validado } \\
\text { (GOHAI) juntamente com } \\
\text { outras variáveis. }\end{array}$ & $\begin{array}{l}\text { Cerca de } 39 \% \text { dos idosos nunca } \\
\text { haviam visitado um dentista e } \\
48 \% \text { eram financeiramente } \\
\text { dependentes. A maioria da } \\
\text { amostra não possuía seguro } \\
\text { saúde de qualquer tipo. Os } \\
\text { entrevistados que realizaram } \\
\text { procedimentos adequados de } \\
\text { higiene oral exibiram pontuações } \\
\text { médias GOHAI mais altas do } \\
\text { que aqueles que não realizaram. } \\
\text { Entrevistados sem problemas } \\
\text { dentários nos últimos } 12 \text { meses } \\
\text { relataram melhor OHRQoL. Foi } \\
\text { ainda reiterado que aqueles com } \\
\text { múltiplos problemas dentários } \\
\text { tiveram pontuações GOHAI } \\
\text { mais baixas. }\end{array}$ \\
\hline $\begin{array}{l}\text { Self-reported oral health } \\
\text { problems and the ability } \\
\text { to organize dental care of } \\
\text { community-dwelling } \\
\text { elderly aged } \geq 75 \text { years, } \\
\text { Bakker et al., } 2020\end{array}$ & $\begin{array}{l}\text { Inglês/ } \\
\text { Holanda }\end{array}$ & $\begin{array}{l}\text { Avaliar problemas de } \\
\text { saúde bucal autorreferidos } \\
\text { (como secura bucal, dor, } \\
\text { problemas de mastigação) } \\
\text { em idosos (idade } \geq 75 \\
\text { anos) que são frágeis ou } \\
\text { têm necessidades } \\
\text { complexas de cuidados. }\end{array}$ & $\begin{array}{l}\text { Delineamento: estudo } \\
\text { transversal. Uma amostra de } \\
3533 \text { idosos participantes do } \\
\text { projeto "Embrace", foram } \\
\text { convidados a preencher } \\
\text { questionários sobre estado } \\
\text { bucal e problemas de saúde } \\
\text { bucal. }\end{array}$ & $\begin{array}{l}\text { Boca seca }(11,7 \%) \text { e dor oral } \\
(6,2 \%) \text { foram os problemas mais } \\
\text { relatados. Idosos com } \\
\text { necessidades complexas de } \\
\text { cuidado relataram a maioria dos } \\
\text { problemas de saúde bucal. Em } \\
\text { caso de um idoso procurar } \\
\text { tratamento odontológico para } \\
\text { aliviar uma queixa de dor bucal, } \\
\text { a maioria dos idosos deste estudo } \\
\text { conseguiu providenciar } \\
\text { atendimento odontológico e } \\
\text { transporte até o dentista. Idosos } \\
\text { frágeis e complexos muitas vezes } \\
\text { precisam da ajuda de cuidadores } \\
\text { para visitar o dentista. }\end{array}$ \\
\hline
\end{tabular}

Fonte: Pesquisa direta (2021).

\section{Discussão}

Cornejo et al. (2013) em seu estudo realizado com o Geriatric Oral Health Assessment Index (GOHAI) e o Oral Health-Related Quality of Life (OHRQoL), apontaram que a população de idosos estudada apresentou uma baixa qualidade de vida relacionada à saúde bucal e uma prevalência de saúde bucal precária, corroborando com os estudos similares conduzidos Zuluaga et al. (2012). Esses índices podem captar os danos causados pela cárie, doença periodontal e edentulismo na terceira 
idade (Cornejo et al., 2013), fatores esses que devem ser a base da atuação do cirurgião-dentista, visando uma melhora da saúde bucal e, consequentemente, da qualidade de vida.

Além disso, os autores destacam que idosos que necessitaram de reabilitações com próteses estivessem com menores índices do que aqueles com dentes naturais, demonstrando a importância do estado clínico da saúde bucal na vida dos mesmos, fazendo com que a atuação do cirurgião-dentista contribuísse para o bem-estar dessas pessoas (Cornejo et al., 2013).

Chahar, Mohanty e Aswini (2019), relatam que valores culturais e sociais são fatores importantes afetando e impactando a percepção de um idoso acerca de sua saúde bucal. Assim, idosos que possuíam uma boa higiene oral exibiram maiores pontuações nos índices utilizados e os que não possuíam problemas dentários nos últimos 12 meses, apresentaram uma melhor qualidade de vida relacionada à saúde bucal (Chahar, Mohanty \& Aswini, 2019).

Os estudos realizados por Heoksema et al. (2017), mostraram que a saúde e higiene bucal dos idosos participantes da pesquisa eram, geralmente, muito precárias, fazendo com que a necessidade de cuidados bucais fosse alta. Além disso, os idosos que possuíam dentes remanescentes se mostraram menos cooperativos em realizar tratamento do que os idosos edêntulos, sendo necessário que os cuidados bucais fossem otimizados, já que muitos desses idosos não eram capazes de realizar sua higiene oral de forma satisfatória e necessitavam dos cuidados de um cirurgião-dentistas.

Bakker et al. (2020) avaliaram a autopercepção dos idosos em relação à saúde bucal, neste estudo observou-se que os problemas mais relatados foram boca seca e dor oral, além disso, os idosos com necessidades complexas relataram a maioria dos problemas relacionados à saúde bucal. Em estudo similar realizado em base domiciliar em uma área de abrangência da Estratégia de Saúde da Família, Nogueira et al. (2017) chegaram à conclusão de que a predominância do edentulismo, uso, necessidade e substituição de prótese dentária denotam a precária condição dos idosos entrevistados, embora tenham relatado ótima ou boa autopercepção de sua saúde bucal.

Northridge et al. (2017), examinaram em seu estudo as experiências de idosos de minoria étnica/racial que frequentam centros para idosos em Manhattan, na cidade de Nova York, em relação ao atendimento recebido em clínicas de escolas de odontologia. Sendo a principal descoberta descrita que a maioria dos participantes relatou experiências positivas com clínicas escolas de Odontologia, relatando ter recebido resultados excelentes, tratamento seguro e atendimento acessível em clínicas de escolas de odontologia. Assim, as escolas de odontologia foram vistas como honestas e confiáveis, em uma época em que as instituições da sociedade americana não eram universalmente respeitadas. Já as experiências negativas não foram expressas universalmente pelos participantes.

Ainda nessa perspectiva, em um relato de experiências, T. Alves et al. (2017), utilizou como amostra, uma população de idosos institucionalizados, com idade entre 60 e 95 anos, no Município de Governador Valadares, Minas Gerais, Brasil, e constatou-se, por parte dos cuidadores, elevada demanda por treinamento específico para exercer cuidados sobre higienização e cuidados com a saúde bucal dos internos. A partir disto, os discentes envolvidos levaram para a instituição atividades lúdicas que fosse possível manter a atenção dos idosos e assim despertar o interesse dos mesmos acerca das orientações em saúde bucal.

Observa-se assim, a importância do conhecimento pelo cirurgião-dentista sobre a autopercepção dos idosos com relação ao seu estado de saúde, como forma de ajudá-los na adesão de comportamentos saudáveis (Nogueira et al., 2017). No entanto, o profissional deve compreender que a autopercepção ou autoavaliação dos idosos com relação à sua saúde bucal é influenciada por fatores sociais, culturais e no conhecimento prévio sobre o conceito de saúde e doença (Martins et al., 2009). Dessa forma, a avaliação e acompanhamento do cirurgião dentista com relação ao paciente idoso deve ser feita de forma individualizada e humanizada.

Conclui-se então que a promoção em saúde bucal se mostrou efetiva suprindo grande parte das carências levantadas, permitindo um acesso facilitado a informações e incentivando o autocuidado pelos internos da instituição e possibilitou ainda, 
agregar o processo de ensino e aprendizado dos discentes envolvidos tendo em vista que houve grande troca de experiências aproximando assim, estes, da realidade dos idosos atendidos por instituições de seu município.

\section{Considerações Finais}

A atuação do cirurgião-dentista se faz extremamente importante nos cuidados bucais de pacientes da terceira idade, já que muitos fatores que a própria senescência causa, como problemas físicos e emocionais, podem levá-los a uma baixa qualidade de vida relacionada à saúde bucal e uma pobre higiene oral. Esses fatores os levam, dessa forma, a necessitar ainda mais de intervenções, visando um maior bem-estar dessas pessoas.

\section{Referências}

Alves, P., da Costa Filho, O. M., dos Santos, M. F. \& Sartorello, R. (2017). A manutenção da capacidade funcional como qualidade de vida no envelhecimento no município de São Paulo-SP. Revista Científica, 1(1).

Alves, T. S., Félix, A. R., Soares, J. L., Magalhães, L. L., Münchow, E. A., \& de Carvalho, R. F. (2017). Relato de experiências educativas em saúde bucal para idosos institucionalizados. Revista Brasileira de Extensão Universitária, 8(3), 167-174.

Ástvaldsdóttir, Á., Boström, A. M., Davidson, T., Gabre, P., Gahnberg, L., Sandborgh Englund, G., \& Nilsson, M. (2018). Oral health and dental care of older persons-A systematic map of systematic reviews. Gerodontology, 35(4), 290-304.

Bakker, M. H., Vissink, A., Spoorenberg, S. L., Wynia, K., \& Visser, A. (2020). Self-reported oral health problems and the ability to organize dental care of community-dwelling elderly aged $\geq 75$ years. BMC Oral Health, 20(1), 1-9.

Brasil, \& Presidência da República. (2003). Lei n ${ }^{\circ} 10.741$, de $1^{\circ}$ de outubro de 2003. Dispõe sobre o Estatuto do Idoso e dá outras providências. Diário Oficial da União.

Castrejón-Pérez, R. C., Borges-Yáñez, S. A., Irigoyen-Camacho, M. E., \& Cruz-Hervert, L. P. (2017). Negative impact of oral health conditions on oral health related quality of life of community dwelling elders in Mexico city, a population based study. Geriatrics \& gerontology international, 17(5), 744-752.

Chahar, P., Mohanty, V. R., \& Aswini, Y. B. (2019). Oral health-related quality of life among elderly patients visiting special clinics in public hospitals in Delhi, India: A cross-sectional study. Indian journal of public health, 63(1), 15.

Cornejo, M., Pérez, G., de Lima, K. C., Casals-Peidro, E., \& Borrell, C. (2013). Oral health-related quality of life in institutionalized elderly in Barcelona (Spain). Medicina oral, patologia oral y cirugia bucal, 18(2), e285.

Donnelly, L. R., Clarke, L. H., Phinney, A., \& MacEntee, M. I. (2016). The impact of oral health on body image and social interactions among elders in longterm care. Gerodontology, 33(4), 480-489.

Glick, M., Williams, D. M., Kleinman, D. V., Vujicic, M., Watt, R. G., \& Weyant, R. J. (2016). A new definition for oral health developed by the FDI World Dental Federation opens the door to a universal definition of oral health. British dental journal, 221(12), $792-793$.

Hoeksema, A. R., Peters, L. L., Raghoebar, G. M., Meijer, H. J., Vissink, A., \& Visser, A. (2017). Oral health status and need for oral care of care-dependent indwelling elderly: from admission to death. Clinical oral investigations, 21(7), 2189-2196.

Hopia, H., Latvala, E., \& Liimatainer, L. (2016). Reviewing the methodology of an integrative review. Nordic College of Caring Science,30, 662-669. https://doi.org/10.1111/scs.12327.

IBGE, C. D. P. (2015). Síntese de indicadores sociais: uma análise das condições de vida da população brasileira: 2015

IBGE-Instituto Brasileiro de Geografia e Estatística. (2013). Projeção da população do Brasil por sexo e idade para o período 2000 a 2060.

Lyu, W., \& Wolinsky, F. D. (2017). The onset of ADL difficulties and changes in health-related quality of life. Health and quality of life outcomes, 15(1), 130 .

Martins, A. M. E. D. B. L., Barreto, S. M., \& Pordeus, I. A. (2009). Auto-avaliação de saúde bucal em idosos: análise com base em modelo multidimensional. Cadernos de Saúde Pública, 25, 421-435.

Masood, M., Newton, T., Bakri, N. N., Khalid, T., \& Masood, Y. (2017). The relationship between oral health and oral health related quality of life among elderly people in United Kingdom. Journal of dentistry, 56, 78-83.

Nogueira, C. M. R., Falcão, L. M. N., Nuto, S. D. A. S., Saintrain, M. V. D. L., \& Vieira-Meyer, A. P. G. F. (2017). Self-perceived oral health among the elderly: a household-based study. Revista Brasileira de Geriatria e Gerontologia, 20, 07-19.

Northridge, M. E., Schenkel, A. B., Birenz, S., Estrada, I., Metcalf, S. S., \& Wolff, M. S. (2017). "You Get Beautiful Teeth Down There": Racial/Ethnic Minority Older Adults' Perspectives on Care at Dental School Clinics. Journal of dental education, 81(11), $1273-1282$.

Raphael, C. (2017). Oral health and aging.World Health Organization. (2015). World report on ageing and health. World Health Organization. 
Research, Society and Development, v. 10, n. 13, e87101321028, 2021

(CC BY 4.0) | ISSN 2525-3409 | DOI: http://dx.doi.org/10.33448/rsd-v10i13.21028

World Health Organization. (2017). Integrated care for older people: guidelines on community-level interventions to manage declines in intrinsic capacity.

Zuluaga, D. J. M., Montoya, J. A. G., Contreras, C. I., \& Herrera, R. R. (2012). Association between oral health, cognitive impairment and oral health-related quality of life. Gerodontology, 29(2), e667-e673. 